\title{
Climate\#driven range shifts reduce persistence of competitors in a perennial plant community
}

\section{Journal Article}

\section{Author(s):}

Usinowicz, Jacob; Levine, Jonathan M.

Publication date:

2021-05

Permanent link:

https://doi.org/10.3929/ethz-b-000491558

Rights / license:

In Copyright - Non-Commercial Use Permitted

Originally published in:

Global Change Biology 27(9), https://doi.org/10.1111/gcb.15517

\section{Funding acknowledgement:}

173210 - Timescales of changing species interactions under warming climate (SNF) 
This is the peer reviewed version of the following article: Usinowicz, J. \& Levine, J.M. (2021). Climate-driven range shifts reduce persistence of competitors in a perennial plant community. Glob. Chang. Biol., 27, 1890-1903., which has been published in final form at https://doi.org/10.1111/gcb.15517. This article may be used for non-commercial purposes in accordance with Wiley Terms and Conditions for Use of Self-Archived Versions. 
Running head: Climate-driven range shifts reduce persistence

1 Climate-driven range shifts reduce persistence of competitors in a perennial plant community

3

Jacob Usinowicz ${ }^{1 *}$ and Jonathan M. Levine ${ }^{2}$

5

$6{ }^{1}$ Department of Zoology and Biodiversity Research Centre, University of British Columbia,

7 Vancouver, Canada, V6T 1Z4.

$8 \quad{ }^{2}$ Department of Ecology and Evolutionary Biology, Princeton University, New Jersey, USA, 08544

$9 *$ *Correspondence to: usinowic@zoology.ubc.ca

10

11 ORCIDs:

12 Jacob Usinowicz: 0000-0003-1397-005X

13 Jonathan M. Levine: 0000-0003-2857-7904

14

15 Classification: Biological Sciences/Ecology

16

Keywords: climate change, range shifts, community ecology, coexistence theory, alpine plants 17

Author contributions: JU and JML conceived the study. JU conducted the experiment with oversight from JML. JU analyzed data with input from JML. JU wrote the original draft of the manuscript, JU and JML wrote and edited subsequent drafts.

This PDF file includes:

23 Abstract: 296 words

24 Main Text: 7450 words

25 Tables: 2

26 Figures: 3

27 Supplementary Information 1-4 are contained in a separate file 
Abstract

Forecasting the impacts of climate change on species persistence in diverse natural communities requires a way to account for indirect effects mediated through species interactions. In particular, we expect species to experience major changes in competition as they track favorable climates. Here, we combine experimental data with a recently developed theoretical framework based on coexistence theory to measure the impact of climate-driven range shifts on alpine plant persistence under climate change. We transplanted three co-dominant alpine perennial species to five elevations, creating a maximum $5^{\circ} \mathrm{C}$ increase in average growing season temperature. We statistically modeled species' demographic rates in response to the environment and interpolated species intrinsic ranges - the environmental mapping of reproduction in the absence of competition. We used low-density population growth rates - species' initial rate of invasion into an established community - as a metric of persistence. Further analysis of low-density growth rates allowed us to parse the direct impacts of climate change from indirect impacts mediated by shifting competition. Our models predict qualitatively different range shifts for each species based on the climate conditions under which growth rates are maximized and where they are zero. Overall, climate change is predicted to increase the intrinsic (competition free) growth rates of all species, as warmer and wetter conditions increase the favorability of alpine habitat. However, these benefits are entirely negated by increased competition arising from greater overlap between competitors in their intrinsic ranges. Species were highly dispersal limited, which can prevent species from tracking shifting intrinsic ranges by reducing population spread rates. Yet dispersal limitation also promoted species' persistence because it promotes persistence mechanisms. Our study demonstrates the complex pathways by which climate change impacts species' persistence by altering their competitive environment, and highlights how a persistence framework based on low-density growth rates can

51 help disentangle impacts. 


\section{Introduction}

Global climate change is rapidly altering the habitat of many species, shifting the location of favorable environments, and exposing species to environments that are novel or even without historical analogues (Radeloff et al., 2015; Williams, Jackson, \& Kutzbach, 2007). Whether climate change threatens species' potential to persist depends on both direct climate impacts on the extent and suitability of habitat (Guisan \& Thuiller, 2005; Wiens, Stralberg, Jongsomjit, Howell, \& Snyder, 2009), and indirect impacts resulting from a reshuffling of species interactions (Alexander, Diez, \& Levine, 2015; Urban, Tewksbury, \& Sheldon, 2012; Zarnetske, Skelly, \& Urban, 2012). Species differences in how their demographic rates respond to changes in the locations of favored habitat (Chen, Hill, Ohlemüller, Roy, \& Thomas, 2011; Hoffmann \& Parsons, 1997; Root et al., 2003) will inevitably cause range shifts that are asynchronous across species (Usinowicz \& Levine, 2018). Because species interactions are a major determinant of habitat suitability (Davis et al., 1998; A. Ettinger \& HilleRisLambers, 2017; A. K. Ettinger \& HilleRisLambers, 2013), the changing interactions that come from reshuffling communities are likely to have a major impact on species persistence (Thompson \& Gonzales, 2017; Alexander, Diez, \& Levine, 2015; Urban, Tewksbury, \& Sheldon, 2012; Zarnetske, Skelly, \& Urban, 2012). Fully accounting for the impacts of climate change on species' persistence thus requires ways to incorporate biotic interactions into a predictive understanding of range shifts.

Although considerable evidence supports the role of species interactions in shaping species ranges today, predicting how changing interactions under climate change will impact persistence requires novel approaches. Given that species' interactions with competitors are known to be a primary determinant of their contemporary geographic distribution and local abundance (HilleRisLambers, Harsch, Ettinger, Ford, \& Theobald, 2013; Sexton, McIntyre, Angert, \& Rice, 2009; Wisz et al., 2013; A. Ettinger \& HilleRisLambers, 2017, Jankowski, Robinson, \& Levey, 2010; Stanton-Geddes, Tiffin, \& Shaw, 2012), a species' ability to persist across its range is likely to be impacted by the new competitive landscape that emerges with climate change (Alexander, Diez, Hart, \& Levine, 2016; Usinowicz and Levine, 2018). In fact, climate-driven shifts in competition may have larger impacts on species persistence than the direct effects of changing climate itself (Alexander, Diez, Hart, \& Levine, 2015; Usinowicz and Levine, 2018). However, tools to predict the consequences of climate-driven range shifts for species persistence in a community-level context remain largely underdeveloped due to conceptual challenges inherent in linking local-scale population dynamic processes and competition to range-scale forecast of species' viability.

Moreover, local-scale demographic measurements relevant to persistence (i.e. birth and death rates) tend to require intensive collection efforts, and the quantitative modeling and analysis of range dynamics can become highly technical when explicitly accounting for dispersal. 
Recently, several authors have suggested that the dynamics of species ranges can be recast as a geographic-scale coexistence problem in ways that advance our understanding of competitionmediated impacts of climate change (Godsoe, Jankowski, Holt, \& Gravel, 2017; Usinowicz \& Levine, 2018). Range dynamics and coexistence theory are naturally linked by several convergent concepts (Godsoe, Jankowski, Holt, \& Gravel, 2017). These include the spatial map of species' intrinsic growth rate response to the abiotic environment, referred to as the "intrinsic range" (Schurr et al., 2012) in the range dynamics literature and the "environmental response" in the coexistence literature (P. Chesson, 2000). In the absence of species interactions and dispersal limitation, range dynamics can be understood entirely from the shifting location of species' intrinsic ranges (Engler et al., 2009). However, competition, dispersal, and species' responses to the environment jointly determine species "realized ranges" and ultimately their ability to persist (Usinowicz \& Levine, 2018). Coexistence theory integrates all of these features into a metric of persistence known as the low-density growth rate (LGR) (Peter Chesson, 2000; Robin E. Snyder \& Chesson, 2004) - the population growth rate of a focal species when it is at near-zero population density, and competitors have been given time to reach an equilibrium in the absence of the focal low-density species. Assuming empirical studies can provide demographic and competitive rates across a species range, then the LGR provides a range-level metric of persistence (Usinowicz \& Levine, 2018).

A range-scale LGR can be used as a metric to quantify species persistence under current and projected range configurations, and a tool to distinguish the direct impacts of climate change from the indirect influence of changing environmental conditions that emerge because of local-scale competition. The LGR can be decomposed into a set of distinct mechanisms that regulate species' realized ranges and thus their persistence- the average range-wide influence of environmental conditions and competition, and a set of distinct spatial persistence mechanisms (Usinowicz \& Levine, 2018; Robin E. Snyder, 2008; Robin E. Snyder \& Chesson, 2004). This decomposition has several advantages. First, separating the average range-wide influence of environmental conditions from the influence of competition allows investigators to separately quantify the direct impacts of climate change from its indirect effects mediated through changes in local-scale competition. Second, the various spatial persistence mechanisms are emergent features of spatial patterns in species' distributions - in particular, the clustering and overlap of competitors - that create additional feedbacks between species' local population dynamics and realized ranges. Because there are only a few generalized mechanisms (i.e. they can be quantified in any spatial population model) that determine how range shifts impact persistence, they facilitate comparison of range-shift scenarios across different communities (Usinowicz \& Levine, 2018).

The range-scale LGR is particularly well suited to evaluating the complicated role that dispersal plays in species’ persistence following climate change. On the one hand, dispersal 
123 limitation reduces population spread rates (Kot, Lewis, \& van den Driessche, 1996; Neubert \& 124 Caswell, 2000) which makes it more likely that species fail to track preferred habitat as climate 125 changes (Brooker, Travis, Clark, \& Dytham, 2007; Guisan \& Thuiller, 2005). On the other hand, 126 dispersal limitation promotes species persistence with competitors when it allows species to concentrate reproductive effort in preferred habitat and avoid competition (Bolker, Pacala, \& Neuhauser, 2003; R. E Snyder \& Chesson, 2003; Robin E. Snyder, 2006). To our knowledge, these trade-offs of dispersal limitation for population spread rates and persistence in a climate change context have not been explored, despite their potential significance for understanding species persistence in a changing environment. The LGR provides an integrated way to account for the effects of local dispersal on range wide persistence because it can be calculated with and without dispersal limitation. Moreover, the LGR is also a key determinant of the population spread, the other being dispersal (Kot, Lewis, \& van den Driessche, 1996; Neubert \& Caswell, 2000). As we demonstrate below, this means that the LGR can be used to separate the benefits of dispersal limitation for species persistence with competitors from the negative impacts of slow population spread rates.

Here, we empirically parameterize a range dynamics framework based on the LGR from coexistence theory (Usinowicz and Levine 2018) to investigate climate-change impacts on alpine plant communities. Many alpine plant species are notably sensitive to climate and have rapidly shifted ranges in response to recent climate change (Alexander et al. 2018; Chen et al. 2011; Lenoir, Gégout, Marquet, De Ruffray, \& Brisse, 2008). They also face a high risk of local extinction because their ranges can be constrained to relatively narrow elevational regions, and because warmer climate is expected to eventually push many species' ranges against upward elevational limits beyond which expansion is impossible (Engler et al., 2011). At the same time, the mechanisms that shape the elevational ranges of alpine plants are the same as those that determine latitudinal ranges of many plant species more generally (Jump, Mátyás, \& Peñuelas, 2009). Thus insights and methodology developed to understand the elevational range dynamics of mountain species can scale up to help understand range dynamics at broader latitudinal scales (Alexander et al., 2018).

Our approach for predicting range dynamics and persistence combines experimental, statistical, and theoretical techniques to the demographic rates of three alpine plant species under bookending climate-change scenarios. More specifically, we build a spatially explicit population model that predicts range shifts and persistence, and parameterize the model with experimental data from field sites in the Swiss Alps. At the core of our experimental approach is a three-year competition experiment replicated at five different elevations that we use to infer environmental drivers of intrinsic growth rates, competition, survival, and dispersal rates. We focus our efforts on 
158 three co-occurring alpine plant species that are broadly representative of major perennial alpine 159 plant types and are three of the most abundant species in our study system, as well as being 160 common throughout the European Alps. We calculate the LGR for these species under climate 161 change to address the following questions: (1) What is the magnitude of climate change impacts that are caused directly by changing environmental conditions relative to those that are mediated via changing competition? (2) How does dispersal limitation influence each of these effects in ways that impact species persistence? (3) Which spatial persistence mechanisms shift most under climate change to influence persistence?

\section{Methods}

168 Our methodology combines multi-year field experiments, statistical analyses, and mathematical 169 modeling to arrive at measures of low-density growth rates (LGRs) for three co-occurring alpine 170 plant species. In what follows, we first describe the study system and experimental design (section, 171 Study system and experimental design). We then detail our mathematical model for plant population growth in the system, the statistical approaches used to fit model parameters to field data, and methods for predicting future parameter values using the statistical models and projected climate conditions (section, Theoretical model of population growth and fitted parameters). Last, we explain how the fully parameterized mathematical models were used to (1) quantify LGRs under current and future climate conditions, (2) analyze the contribution of spatial persistence mechanisms to LGRs, and (3) investigate the role of dispersal limitation in determining persistence (section, Analysis of persistence and spatial mechanisms).

\section{Study system and experimental design}

We conducted a large-scale experiment to measure the impacts of changing climate on the fitness and population dynamics of several plant species, in the presence and absence of competition from one another. We conducted the experiment along the Calanda mountainside in the Swiss Alps (Canton des Grisons), which is typical of moist, temperate, calcareous mountain environments. For our experiment, we selected three alpine plants species: Dactylis glomerata, Alchemilla xanthocholra, and Helianthemum nummulerium in accordance with three criteria. First, these three species were amongst the most abundant plant species at the study site, and therefore most important for overall community dynamics. Their relative abundance was determined through preliminary transect surveys conducted in spring 2014, where relative ground cover was assessed in $1901 \mathrm{~m}^{2}$ quadrats over the altitudinal range of $1000-2000 \mathrm{~m}$. Second, these species were readily found in dense clusters that could be easily manipulated to form experimental monocultures. Singlespecies monocultures are required for measuring a competitor's LGRs in a system where the 
background "resident" competitor is at a constant, near equilibrium density. Finally, each species is broadly representative of more general plant types commonly found in alpine meadows: Dactylis is a fast-growing grass species; Alchemilla is typical of moderately-fast growing alpine forbs with moisture and nutrient demands similar to Dactylis; and Helianthemum is typical of many alpine species with prostrate evergreen shrub growth forms, and is more tolerant of the drier and poorer soils common in mountains.

We measured the influence of climate on species' reproductive fitness and survival by transplanting individuals that originated from a small region near $1900 \mathrm{~m}$ asl to five other sites at elevations of 1000,1400,1600,1800,2000m asl along the same mountainside. These sites are no more than $1.5 \mathrm{~km}$ apart and have similar southeast exposure, aspect and calcareous bedrock.

In September of 2014, we excavated individuals of each species from sites where it was highly abundant. Within a week of removal, individuals of all three species were transplanted into a bare soil treatment (10 per site), where all other vegetative biomass had been removed, and into single-species competition treatments (10 per "background competitor" per site). The single species competitive "backgrounds: were established as follows. In September of 2014, roughly $30 \mathrm{~cm}$ diameter turfs with $>80 \%$ cover of one of focal species were excavated to $20 \mathrm{~cm}$ depth and transplanted to each of the five sites. To control for local adaptation and other potentially confounding factors, these turfs were all sourced from the same restricted region at $1900 \mathrm{~m}$ asl that sourced our focal individuals. Each site received 10 replicates of each focal species dominated turf, which were weeded to a monoculture in April of 2015, and kept in this state through the duration of the experiment. By placed a single transplanted individual of each of the three focal species into each of the monoculture turfs (in fall 2014), we mirrored the theoretical measurement of local LGRs described below. Specifically, we allowed a low density of individuals (i.e. the population of focal species individuals replicated at a specific elevation) to invade a resident at equilibrium density (i.e. the established background turfs).

Only H. nummularium focal individuals showed significant transplant mortality after the winter of 2014-2015, so dead individuals were replaced in April 2015. Each growing season from 2015 to 2017, we recorded the number of flowers (for A. xanthochlora, and H. nummularium) or the size of inflorescences (for D. glomerata). For Alchemilla and Helianthemum, flower counts were converted directly to seed counts since each flower produces a single seed. Dactylis inflorescence size was converted to seed counts using an allometric scaling relationship calculated from several published relationships between inflorescence size and seed number for this species (Tormo-Molina et al. 2015; Borawska-Jarmulowicz 2018 ). At the end of each summer we recorded the survival of all transplants, and the height of the inflorescences for use in dispersal modeling. 
The demographic responses of the plants were related to climate variables measured on site and through remotely sensed data. We used HOBO pendant loggers to measure temperature and solar irradiation at each site. Beginning in June 2015, three loggers were placed per site at the soil surface, two on bare soil treatments and one within vegetation at plot margins. Loggers were swapped every three months for maintenance and to retrieve time-series of temperature and solar irradiance at soil level.

We used publicly available remotely sensed data to obtain measures of soil moisture deficit (Terraclimate: Abatzoglou, Dobrowski, Parks, \& Hegewisch, 2018). Soil moisture variables were retrieved from Terraclimate remote sensing layers based at $4 \mathrm{~km}^{2}$ grids; when projected onto our sites this created 4 unique time series corresponding to our sites (the $1600 \mathrm{~m}$ and $2000 \mathrm{~m}$ sites are within the same grid cell).

The spatial scale of the measured environmental variables was much coarser than what we required to model population dynamics. Therefore we projected these coarse-scale measurements to a finer $\left(1 \mathrm{~m}^{2}\right.$ grid cell) scale. To do this, we first fit the gradient in each environmental variable across our five study sites using a GAMM with elevation as the only predictor variable and year as a random effect, and then used the fitted GAMMs to interpolate environmental measures at the finer spatial resolution (holding the year effect at its average).

\section{Theoretical model of population growth : mathematical structure and parameter fitting}

In order to model the dynamics of competing species living along a climate gradient, we adapted the discrete time Leslie-Gower model of competition to perennial plants living in a linear series of adjacent sites, following Usinowicz and Levine (2018). We summarize the important features of the model here but refer the reader to Supporting Information 1, and Usinowicz and Levine (2018) for complete details. In the model, plant populations grow, compete, and disperse along a climate gradient determining species' intrinsic fecundity. All individuals survive at a species-specific, spatially and temporally constant annual rate. Potential reproduction, by contrast, varies across the environmental gradient, and is species-specific. Here, we use General Additive Models (GAMs) to describe the environmental mapping of intrinsic reproduction with respect to elevation for each focal species. Intrinsic reproduction is reduced by interspecific and intraspecific competition and the resulting seeds are dispersed according to species-specific dispersal kernels (see below). Combining these demographic processes leads to the following integro-difference equation (see also equation S1.1) for population dynamics:

$$
n_{j}(x, t+1)=\int_{-\infty}^{\infty} k_{j}(x-y) \frac{E_{j}(y, t)}{C_{j}} n_{j}(y, t) d y+s_{j} n_{j}(x, t) .
$$


Here, $n_{j}$ is the population size of species $j$ at location $x$ at generation $t$. The relationship between species' demographic response and the environment at all locations $x$ is defined by the per-capita intrinsic fecundity $E_{j}(x, t)$. It is a species-specific, location-specific fecundity that is determined by the suitability of the environment (measured in fecundity units) at a particular location and time. Seeds disperse according to a dispersal kernel $k_{j}(x-y)$, which gives the probability a seed travels distance $x$ - $y$. Adults survive at a density-independent, spatially homogenous rate $s_{j}$. Competition from conspecific and heterospecific individuals reduces $E_{j}(x, t)$ according to $C_{j}$, which incorporates the fact that closer neighbors have a stronger effect than those further away (see eq S1.2 for details of $C_{j}$ ). Equation 1 is the model we parameterize with the field data described in the prior section, and ultimately allows us to calculate a LGR for each of our focal species, explained below in the section "Analysis of persistence and spatial mechanisms." We next explain how we fit the key elements of the model with the field data.

\section{Fitting spatially-independent parameters $C_{j}$ and $s_{j}$}

The total competition $C_{j}$ includes competition coefficients that describe the per-capita rates of competitive suppression between all pairs of species (eq S1.2). We inferred pairwise competition coefficients from our experimental data by first reducing the full spatial model to a local, turf-level model without any spatial variation or dispersal; that is, the model only includes species' average local intrinsic growth rates and pairwise interaction coefficients (S2.1 and S2.2). We then used nonlinear least squares (NLS) to fit inter- and intraspecific competition by comparing the growth of transplanted focal individuals against each competitive background type. Competitive effects fit in this way are still a combination of a per-capita interaction rate and the background density of competitors (i.e. the product of competition coefficients and background competitor density, $\alpha_{i r}$ $n_{r}(x, t)$ in S2.1). Standard per-capita competition coefficients were obtained by dividing each fitted interspecific competitive effect by the fitted intraspecific competitive effect (i.e. S2.3) of the corresponding background species. This divides out the resident background density $\left(n_{r}(x, t)\right)$ and creates a relative competition coefficient, where all competitive is measured as a ratio of interspecific to intraspecific competitive effects. This relative interspecific competition coefficient was used in the full spatial model. See Supplementary Information 2 (Section: Relative competition coefficients) for more details.

Survival $s_{j}$ was determined from the data on annual focal survival (binary response) across all treatments and sites. We fit species-specific general additive mixed-models (GAMMs) (Wood, 2006) with a logit-link function testing for the fixed effects of treatment (background species) and site, and random effect of year. Since these analyses revealed only slight spatial variation in survival, we used the overall mean of each model as a spatially-independent $s_{j}$. 
296 Fitting current and future species' reproductive response to the environment $E_{j}(x, t)$

297 Species' current intrinsic ranges - the mapping of their intrinsic growth rates to environmental conditions - were statistically modeled from species' seed production in the absence of competition across all sites. Although the intrinsic growth rate is a function of all demographic variables, only species' reproductive response to the environment $E_{j}(x, t)$ was assumed to vary with climate, so our use of intrinsic range in what follows only concerns reproduction. We fit GAMMs (Wood, 2006) to explain reproduction using different combinations of mean and minimum growing season temperature, total growing-degree days (GDD), and soil moisture deficit as predictor variables. These abiotic variables were chosen because they were representative of the three main classes of environmental drivers - solar irradiance, moisture, and temperature - identified by Austin \& Van Niel (2011) in a review of published SDMs, and because the data were easy to record locally and readily available from remote sensing databases. In all cases the year of observations was considered as a random effect. Details about modeling and model fits are given in Supplementary Information 3.

Species' intrinsic reproduction across the elevation gradient was statistically modeled as two separate processes: probability of flowering, and the number of seeds produced if flowering occurred. Probability of reproduction was fit using GAMMs with year as a random effect and a logit link function. Seed production was also fit using GAMMs with year as a random effect and a negative binomial link function. Smooth terms were constrained such that reproduction was zero at upper $(3500 \mathrm{~m})$ and lower $(0 \mathrm{~m})$ margins; this reduced the potential for overfitting by ensuring that the resulting intrinsic ranges were "closed" at both range margins (SI 3). The best-fit of the candidate models across all combinations of predictor variables was then chosen for each species according to the Akaike information criterion (AIC) (Akaike, 1973). See SI 3 for further details and fits of smooth terms.

Species intrinsic ranges under their current climate were interpolated at a finer $1 \mathrm{~m}$ spatial resolution. This was done by first applying each species' GAMM for probability of flowering and number of seeds to the spatially interpolated gradients of abiotic variables (see Methods, Study system and experimental design). This produced two projections - one for probability of flowering and one for seed production - that were multiplied together to give the intrinsic range based on reproduction. Prediction intervals were generated for these intrinsic ranges by randomly generating 1000 posterior draws of each species' fitted model (using the mean and standard error of the fitted coefficients and their covariance matrix, from each underlying species-specific GAMM for probability of flowering and seed production) and calculating the $95 \%$ intervals of the resulting distribution of posterior simulations. 
Species' future intrinsic ranges were predicted from the fitted GAMMs using projected climate conditions from IPCC Representative Concentration Pathways (RCP). The warming scenarios RCP 2.6 and RCP 8.5 are combined global change model projections that represent the best and worst-case greenhouse gas emission scenarios, respectively. We refer the reader to IPCC (2014) for details of the global change models used to produce data sets, and here we only describe the qualitative climate projections that are relevant to our study. RCP 8.5 predicts the maximum net increase in mean and minimum temperatures with no temperature stabilization by 2100 , while RCP 2.6 predicts a rapid stabilization and low net increase. In our sites, projected moisture availability stays approximately constant in RCP 2.6, but decreases under RCP 8.5. The RCPs do not project changes in growing degree days (GDD), one of the predictor variables in our GAMMs, but GDD can be predicted with changes forecast under the different RCPs. Specifically, we projected future GDD by first fitting the statistical relationships between GDD and mean and minimum temperatures under current conditions at our sites (HOBO logger data), and then using this fitted model to project GDD based on the future RCP projections of mean and minimum temperature. Each set of projected climate conditions thus presents a new spatial environmental gradient that was used to predict species' intrinsic ranges (with prediction intervals) according to the procedure described in the previous paragraph.

\section{Fitted dispersal kernels: the WALD approach}

349 Given that range dynamics also depend on species dispersal capacities, given as $k_{j}(x-y)$ in the 350 mathematical model of population growth (equation X), we estimated species-specific dispersal kernels. We followed a mechanistic analytical approach known as the Wald analytical long-distance dispersal (WALD) kernel (Katul et al. 2005). The WALD kernel was designed to account for the probability of seed canopy escape and the resulting potential for long-distance dispersal events that result from wind flow in and above the canopy. It is given by an inverse-Gaussian distribution with parameters where the probability $p$ of dispersing distance $x$ is:

$$
p(x)=\left(\frac{\lambda}{2 \pi x^{3}}\right)^{1 / 2} \exp \left[\frac{\lambda(x-\mu)^{2}}{2 \mu^{2} x}\right]
$$

$\lambda=(h / \sigma)^{2}$ and $\mu=(h \bar{U} / V)$ where $h$ is the release height of seeds, $\sigma$ and $\bar{U}$ are the variance and mean, respectively of horizontal wind flow, and $V$ is seed terminal velocity. This model thus requires measuring only four parameters (h, $V, \sigma$ and $\bar{U}$ ) (Katul et al., 2005). Seed release height was assumed to be the height of reproductive structures measured in the field. Terminal velocities were taken from the LEDA Traitbase (Kleyer et al. 2008). Wind flow at each of our sites' was 
measured with 2D sonic anemometers placed $1 \mathrm{~m}$ above canopy, and canopy flow characteristics derived from common assumptions about herbaceous meadow flow characteristics from other studies (Katul et al., 2005; Kull \& Zobel, 1991; Massman \& Weil, 1999; Su, Schmugge, Kustas, \& Massman, 2001). Detailed descriptions of parameters in equation 2 and the techniques used to derive their numerical values are given in SI 2 (Section: Dispersal Kernels).

\section{Analysis of persistence and spatial mechanisms}

The LGR in a spatially variable environment predicts persistence analogous to the non-spatial LGR of an invader attempting to establish in a system dominated by an established resident. However, a spatial LGR further requires that the invader species' absolute abundance is near zero while dispersal limitation has concentrated its relative abundance into regions of favorable environmental conditions (Chesson, 2000; Snyder \& Chesson, 2004). The resulting stationary spatial distribution of relative abundance can be operationalized as an iterative process (Shoemaker \& Melbourne, 2016; Usinowicz \& Levine, 2018) where: 1) invasion initiates with a near-zero abundance, 2) a generation of population growth and reproduction proceeds across space, subject to spatial heterogeneity, interspecific competition, and dispersal limitation, 3) the resulting next generation of abundance across space is reduced back to the invasion-level abundance without changing the relative abundance of individuals across space, 4) these steps are repeated until relative abundance across space remains unchanged between successive generations. The stationary low-density distribution therefore reflects the spatial locations that are most favorable to population growth, as affected by abiotic preferences, avoidance of interspecific competition, and dispersal limitation.

Once the stationary low density distribution is reached, the spatial LGR can be calculated following a final generation of population growth.

The spatial LGR can be decomposed into spatial persistence mechanisms which reveal the impacts of particular spatial arrangements of species' ranges on persistence (Usinowicz \& Levine, 2018). These quantities are useful in a practical sense for calculating persistence, but also have biological interpretations that highlight the relative importance of different demographic processes for persistence (Usinowicz \& Levine, 2018; Snyder and Chesson 2004). The first mechanism that we calculated was a benefit from competitor aggregation- the benefit a species gains from having competitors' with a narrowly distributed (rather than evenly distributed) range. It is proportional to the spatial variance of competition exerted by all competitors on a focal species (e.g. equation 4 in Box 1 of Usinowicz and Levine 2018). The second mechanism is a benefit of competitor segregation- the benefits of being spatially offset from competitors in peak fitness and from segregating from competitors with limited dispersal. It is proportional to a sum of two terms which generally correspond to the covariance between a species' fitness and the reduction in fitness 
resulting from competition (e.g. equation 4 in Box 1 of Usinowicz and Levine 2018). Dispersal is inherently factored into this mechanism based on how it segregates an invader species' from residents when these species have different intrinsic ranges. Persistence is maximized when species have their highest intrinsic growth rates in range locations separate from where they experience the greatest impacts of interspecific competition; a benefit augmented when limited dispersal concentrates the focal species' in the regions where its intrinsic growth rates are high relative to the impacts of competitors. Following, Usinowicz and Levine_(2018), we visualized these two mechanisms on a graph where the $\mathrm{x}$-axis measures the benefit of spatial segregation and the $\mathrm{y}$-axis measures the benefit of aggregation. Change in either mechanism was represented as a vector drawn between starting (current conditions) and ending (future conditions) values of both mechanisms.

For both LGRs and spatial persistence mechanisms, we implemented a bootstrap approach to assess uncertainty in predictions. For each set of the posterior draws of species' intrinsic ranges (see previous section, Fitting current and future species'reproductive response...), we generated the corresponding measures of LGRs and spatial persistence mechanisms. This provided a bootstrapped distribution of 1000 samples for each species from which we obtained the $95 \%$ prediction intervals (PIs). Thus these PIs simultaneously encompass uncertainty associated with the underlying statistical fits of all three species' intrinsic ranges, their projection to a finer spatial grid, and the variety of possible persistence outcomes generated when all three species are considered at once.

\section{Population spread rates}

With knowledge of species specific survival rates, dispersal, sensitivity to competition, and relationships between environmental variables and reproduction, one could parameterize our model and predict the LGR for our three focal species under the current or future climate. However, whether species actually reach their suitable locations in the future, and therefore their persistence, also depends on their population spread rate. We therefore performed a second set of numerical simulations where we used the population spread rates to determine whether populations can successfully track their intrinsic ranges on a yearly basis. We calculated the asymptotic population spread rate $c_{j}$ with the equation (Weinberger 1978; Kot et al. 1996; Neubert \& Caswell 2000; Usinowicz \& Levine 2018):

$$
c_{j}=\min \frac{1}{S} \ln (L G R * m(s)) .
$$

The term $m(s)$ relates to the dispersal kernel and is the sum of the moment-generating functions for each component of the population growth equation (i.e. reproduction and survival) (see Usinowicz

429 \& Levine 2018). Because the WALD kernel is not in the family of kernels with exponentially bounded tailed suitable for equation 2, we approximate each species' WALD dispersal kernel with 
an exponential dispersal kernel whose mean and distance at which $90 \%$ of seeds have fallen are approximately equal to the WALD kernel. These exponential approximations to the WALD kernel were only used in the limited population spread rate scenarios.

To account for limited spread, at each time-step, we found the furthest forward individual in each species' realized range (where density was above an arbitrary numerical threshold of population density $=10^{-7}$ ). We then allowed the population to advance a distance $c_{j}$ from this point bounding the region species were allowed to equilibrate or invade. The LGR and other quantities were then calculated on an annual basis using this new truncated intrinsic range. For all projected range shifts, we calculated species' average LGR in the absence of competitors and compared this to their LGRs in the presence of competitors.

All R code used for analyses of intrinsic ranges, range shifts, and persistence, is available at https://github.com/jusinowicz/calanda_range shifts.

\section{Results}

Species' intrinsic reproduction rates were best modeled with the average growing-season temperature and soil-moisture deficit for their site, with a random effect of year (Table 2, Fig.S3.1, Table S3.1). In some cases the number of growing-degree days and the minimum temperature were retained as important explanatory variables. When we used these fitted relationships to interpolate contemporary intrinsic ranges (Fig 1 C-D, solid lines, Austin \& Van Niel, 2011), the three species showed high overlap in their most favored habitat, with Alchemilla growing best at higher elevations.

Assuming IPCC RCP 8.5 (extreme change) or RCP 2.6 (conservative change) (Fig 1A-D, dashed lines) affected the magnitude of the intrinsic range shift, though in both cases, the qualitative results involved increases in peak fecundity for Alchemilla, upslope expansion with a slight decrease in peak fecundity for Helianthemum, and a net translocation for Dactylus. These particular intrinsic range shifts stem from differences in species' responses to multiple environmental variables; this is further evidenced by the fact that asynchrony in intrinsic range-shifts was less dramatic when only two variables (mean temperature and soil moisture) were used as predictors (Supplementary Information 4, Figure S4.1, Table S4.3).

When species' projected intrinsic ranges were incorporated into the full population dynamic model with survival, competition, and dispersal, their realized ranges (Fig S4.2 ) largely mirrored intrinsic ranges. In part, this reflected that fact that while interspecific and intraspecific competition were both strong, intraspecific competition was typically greater (Table 1), causing species to be somewhat autonomous. Both survival and competition varied across elevation but this variation was relatively small and thus we chose to use the average values in our analysis of persistence. Dispersal 
also varied with elevation following the response of vegetation height. However, the influence of dispersal variation was beyond the scope of the current study and thus average plant heights were used in calculations of LGR reported here.

For all projected range shifts, species' average LGRs increased with climate change in the absence of competition (Figure 1 A, B), following the changes in species' intrinsic reproduction (Figure A-B). However, for all three taxa, these benefits of climate change reversed in the presence of competitors also responding to altered climate conditions. That is, the LGR decreased under climate change for Alchemilla, Dactylis, and Helianthemum (Figure 1 E-F). The magnitude of the impact of climate change on the LGR depended to some degree on the way that GAMMs modeled the relationship between species' intrinsic ranges and environmental variables (SI4, Table S4.2). Prediction intervals (PIs) were large relative to the means, reflecting the combined sources of uncertainty from GAMM coefficient estimates, intrinsic range projections, and variation in competition emerging from different spatial arrangements of competitors. However, the bottom PI of each LGR forecast for Dactylis and Alchemilla was above the threshold for persistence in all cases (LGR > 1), while the upper PI was always below the persistence threshold for Helianthemum.

Decomposing the LGR into spatial persistence mechanisms showed that the spatial distribution of competitors has a major influence on how climate change affects species' persistence (Figure 2). For all three species, the benefit to persistence from competitor aggregation (the spatial clustering of a species' competitors; $y$-axis Figure 2) declined with climate change. For Alchemilla and Dactylis the benefit of competitor segregation declined substantially (the spatial offset of competitor intrinsic ranges; $x$-axis Figure 2). Overall, climate change had the largest impact on persistence via the benefits of competitor segregation. Dactylis and Alchemilla both expanded their intrinsic ranges up-slope, however Dactylis expanded faster which ultimately increased their pairwise overlap in space (Figure $1 \mathrm{C}$-D; 60-year projection vs. 0 -year projection). Helianthemum was also harmed by this increasing range overlap with the other competitors (Figure 1 E-F) but nonetheless experienced a slight benefit of competitor segregation overall, likely due to its decreasing overlap with Alchemilla. In 60 years, the realized range of Helianthemum under RCP 8.5 (Figure $1 \mathrm{G}$ ) is projected to fall almost entirely outside of Alchemilla's. At the same time, the benefit of aggregation for Helianthemum decreased because both of its competitors spatially expanded their ranges. We found only small effects of climate change on the average competitive ability of the species. Again, prediction intervals on the persistence mechanisms were large and reflect the sensitivity of spatial persistent mechanisms to changes in community-level spatial pattern.

All three species dispersed very short distances on average- mean dispersal distance was predicted to be $0.22 \mathrm{~m}, 0.04 \mathrm{~m}$, and $0.03 \mathrm{~m}$ for Dactylis, Alchemilla, and Helianthemum, 
respectively. However, the WALD kernel reveals that turbulent wind patterns can still move $10 \%$ of seeds further than $1 \mathrm{~m}$ for all three species given their small seed size (SI 2). The WALD kernels do not factor in active dispersal mechanisms such as transport by foraging animals (e.g. ants and grazing ungulates). Thus, additional rare long-distance dispersal events may be underestimated by our approach, but little is known about the potential of our focal species to disperse by these means.

The low mean species-specific dispersal distances increased species' LGRs relative to cases where range-wide dispersal was allowed (Figure 3, dark vs. intermediate shading for all species). For Helianthemum, global dispersal across its range causes its LGR to drop below 1, meaning that limited dispersal is essential for its persistence. Dispersal limitation will also constrain spread along the altitudinal gradient, potentially preventing species from occupying the full range of habitat available to them. The average population spread rates $c_{j}$ (eq 2) under current conditions were 0.66 , 0.65, and $0.60 \mathrm{~m} / \mathrm{yr}$ for Dactylis, Alchemilla, and Helianthemum respectively. For comparison, species current average temperature conditions move approximately $8 \mathrm{~m}$ up-slope per year, based on a local increase of $0.044^{\circ} \mathrm{C} / \mathrm{yr}$ in our study site projected by RCP 8.5 (and the initial portion of RCP 2.6). When these limited population spread rates were factored into calculations of LGRs, reproductive output at the leading range edge decreased. A reduction in a focal species' fecundity at the leading range edge sometimes increased itsspecies via spatial persistence mechanisms, as for Alchemilla under RCP 8.5 in years 20 and 60 (Figure 3C, light shading vs. intermediate shading). In other cases, loss of fecundity at the leading range edge reduced the LGR relative to cases where limited dispersal allows populations to segregate but does not constrain spread (Figure 3).

\section{Discussion}

Our results forecast three different types of intrinsic range shift in response to changing climate for the focal alpine meadow species of this study (Fig 1). These included a range expansion (Dactylis), range expansion and translocation with an overall increase in peak intrinsic fitness (Alchemilla), and range expansion with an overall decrease in peak intrinsic fitness (Helianthemum). Competitors strongly regulated the effects of climate-driven range shifts on species persistence, driving the negative effects of climate change on the persistence of two of the three focal species. In fact, in the absence of interspecific competition, climate change increased all species' range-wide LGR by increasing reproduction on average across space (Figure 1 A-B), a result very different than found in the presence of competition (Figure $1 \mathrm{E}-\mathrm{F}$ ). While range expansions and increases in peak fitness were forecast for all species in the absence of competition, species' persistence suffered with climate change in the presence of competitors. By decomposing the LGR into spatial mechanisms of persistence, we showed quantitatively that this is mainly driven by their increasing range overlap with changing climate (Fig 2). While there was potential for 
Helianthemum, to benefit from a partial release from competition at the lower range edge of the community (Fig S4.2), its projected intrinsic range still overlapped too much with the other two competitors for this benefit to emerge. Importantly, while the range of 95\% PIs was large relative to the means, they always encompassed the qualitative forecasts that Dactylis and Alchemilla, are likely to persist under climate change but Helianthemum is not.

Limited dispersal played an important and sometimes contradictory role in determining species' persistence during climate-driven range shifts. On the one hand, dispersal limitation favored species persistence since its removal (i.e. promoting range-wide dispersal) removed all spatial separation of competitors and caused the LGR of Helianthemum to drop below 1, signaling that it can no longer persist in this community (Figure 3 E-F, Table S4.1). However, dispersal limitation also slows population spread rates, and this can reduce persistence (Figure 3, light bars). Overall, however our results suggest that dispersal limitation has some benefits for preserving diversity. This finding greatly complicates the planning of natural reserves by suggesting that natural corridors designed to link habitat patches and aid movement of species at broad scales (Gilbert-Norton, Wilson, Stevens, \& Beard, 2010; Hilty, Lidicker Jr, \& Merenlender, 2012) can have the unintended consequence of reducing species' persistence if they aid the movement of competitively superior species (Haddad et al., 2014; Resasco et al., 2014). For Helianthemum for example, promoting dispersal could help it track preferred environmental conditions, but ultimately threatens its persistence by removing any benefits of spatial aggregation or segregation.

Spatial persistence mechanisms - the benefits of aggregated competitors, and segregation from those aggregated competitors - contributed substantially to species' LGR. Nonetheless two out of three species (Alchemilla and Dactylis) are able to persist even in the absence of any spatial mechanisms, owing to greater intra than interspecific interactions with one another and only Helianthemum went extinct without the spatial segregation provided by dispersal limitation (Fig 3, Table S4.1). These results support results from Adler et al. (2010) that multiple mechanisms of coexistence operate simultaneously in ways that make the coexistence of perennial plants robust. However, the fact that this was not the case for the weakest competitor Helianthemum, supports the notion that competitive rank may predict which species are most sensitive to the climate-driven range shifts in their competitors.

The competition experiment replicated across species' elevational ranges employed here has a number of advantages and disadvantages relative to alternative approaches. Our approach, when coupled to the theoretical framework, allows rather detailed predictions of community dynamics with climate change without eco-physiological understanding of species' environmental responses. However, compared to analyzing observational data with statistical or theoretical models, the 
experimental approach here is considerably more demanding in terms of data collection, and may only be feasible for subunits of a larger community, as done here.

\section{Limitations and Uncertainties}

Our forecasts of climate change impacts on species persistence were sensitive to uncertainties in our statistical models of species' intrinsic ranges (Fig. 1 and 2). The 95\% prediction intervals (PIs) for species' intrinsic ranges show the large range of values that can be produced given the aggregate uncertainty associated with each of the fitted coefficients in the GAMMs (Fig 1. C-D; Fig S4.1 C-D). This uncertainty reflects a major challenge inherent in any field study aiming to predict species' fitness from a complicated multivariate environmental space. As we found in our study, this is especially true when certain combinations of predictor variables (i.e. temperature, soil moisture, and solar irradiation) are poorly sampled simply because they do not commonly occur within the spatial and temporal bounds of the study. Moreover, given that predictions of LGR and spatial persistence mechanisms depend on both the shapes and overlap of species' intrinsic ranges, the 95\% PIs on LGRs (Fig 1. A-B, E-F; Fig S4.1 A-B, E-F) and spatial persistence mechanisms (Fig 2) reflect an additional level of uncertainty associated with how multiple species respond to climate change relative to one another. Though the large PIs associated with our LGRs and measures of spatial persistence mechanisms certainly qualify any conclusions we can draw here, we do believe that being transparent about these uncertainties is critical, as they reflect our true understanding of current and future communities, and the challenges of prediction in this realm.

Further uncertainty in predictions of LGR and spatial persistence mechanisms arises from uncertainty at the level of model selection. Statistical models of climate predictors of demographic rates that might be similar in terms of best-fit metrics or explanatory power could generate very different LGRs when their projections of intrinsic range dynamics differ; this will be most likely to happen when statistical models or fitting procedures select for different numbers or types of explanatory environmental variables. In our study, intrinsic ranges predicted by GAMMs that only allowed for mean temperature and soil moisture deficit as explanatory variables projected slightly different intrinsic ranges and forecasts of LGR (Fig S4.1, Table S4.2) than the results presented in the main text based on more complex GAMMs (Fig. 1).

Last, the application of the persistence framework to analyze experiment results requires making a number of assumptions, especially in selecting the mathematical model of population dynamics and its parameterization, and the selection of focal species. Here, we assumed that species' survival was constant across space and time, independent of environmental conditions, and density-independent, even though environmentally driven variation in survival can influence LGRs (Chesson 2000B). Nor did the population growth rate allow for species specific rates of recruitment 
605 following reproduction. Nonetheless, the theoretical framework is flexible enough to include all of 606 these factors. Finally, our choice of focal species was made to represent the dominant plant types 607 found in our alpine plant community. However, without further experiments involving additional 608 species, it is impossible to know the extent to which our simplified community dynamics capture 609 the full community dynamics of competitors tracking a shifting intrinsic ranges with climate 610 change.

Conclusions

Forecasting the impacts of climate change on diverse competitive communities is currently one of the greatest challenges for ecologists. Here, we have shown that competitive interactions can mediate the major impacts of climate change on species persistence that cannot be forecast from changing habitat suitability alone. Importantly, many of these impacts are unpredictable without considering demography in a spatially explicit framework, and accounting for the interaction between population dynamics and dispersal limitation. In particular, our work suggests that the climate-driven overlap of competitors' ranges can far overwhelm climate-driven increases in species' intrinsic reproductive rates; meanwhile, dispersal limitation can be essential to mitigating increased competition. These inferences are likely to be general for plant communities because all plants are spatially restricted in their ranges, compete locally, and have limited dispersal. The generality of these features suggests that a framework such as the one applied here may provide a way to link local competition outcomes to broader geographical or latitudinal scale forecasts of climate change impacts in many other plant communities.

Acknowledgements. The authors thank Jake M. Alexander for help with experimental design and implementation, Marc-Jacques Mächler for assistance managing summer field crews and data collection, and Regina Maria Zäch and Annette Bieger Altermatt for plant identification. We also thank Werner Eugster for technical insight and guidance with sonic anemometers. 
Running head: Climate-driven range shifts reduce persistence

632 Tables:

633 Table 1. Average values of survival, standardized competition coefficients, and horizontal 634 windspeed for the population models and dispersal kernels. The spatial variation in intrinsic fitness 635 (i.e. species' intrinsic ranges) is plotted in Figure 1A.

636

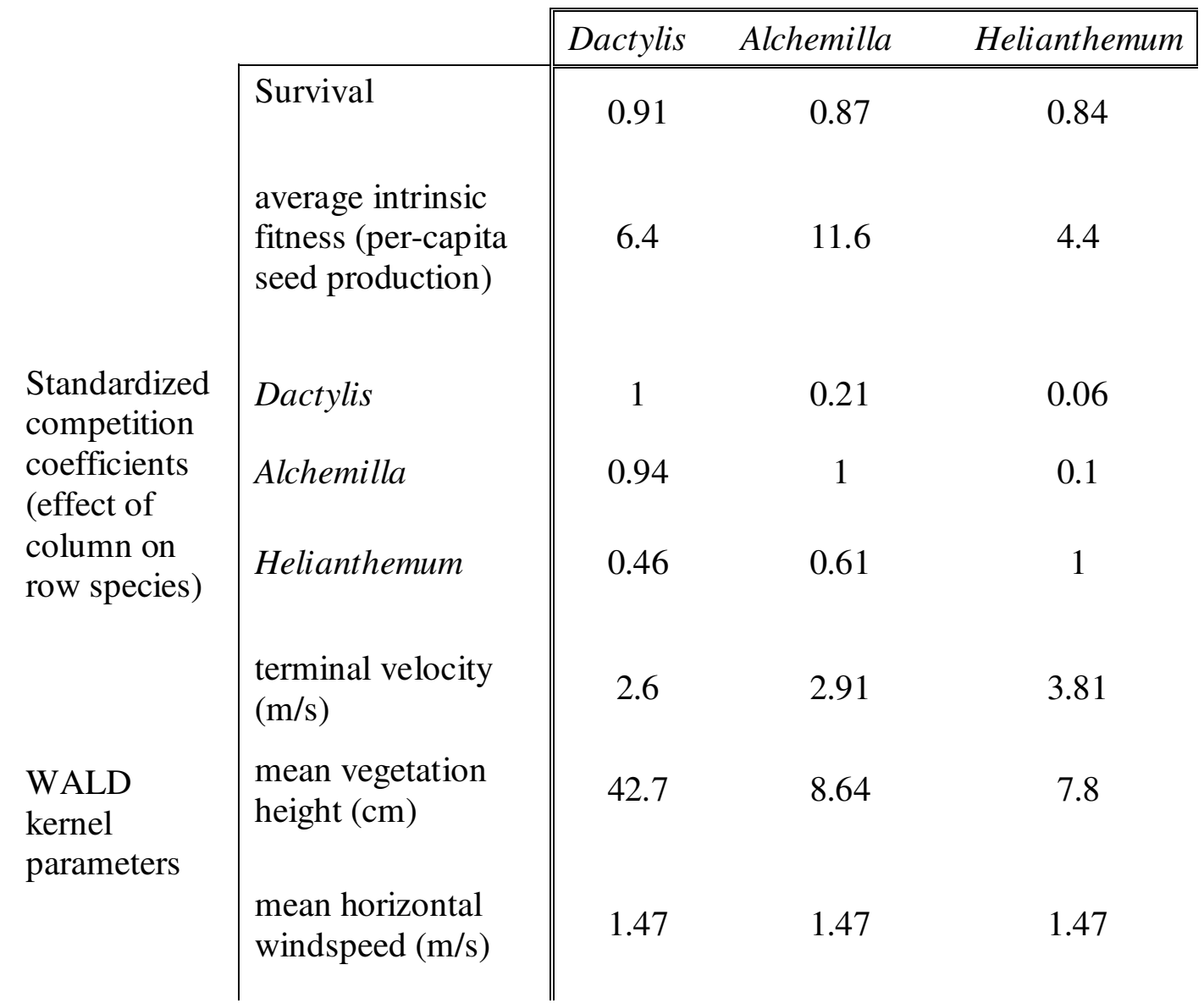


Running head: Climate-driven range shifts reduce persistence

639

640

641

642

643

644

645

646

647

648

649

Table 2. Best-fit statistical models for species' intrinsic reproduction. The probability of reproduction, and number of seeds produced given reproduction were fit separately by general additive mixed models (GAMMs), with the probability of flowering modeled as a binomial process. The predictor variables were all smooth functions (see the main text for more details and SI 3 for plots of each species-specific smooth term). Year was included in every model as a random effect. GDD is growing degree days. The effective degrees of freedom (edf) indicate the complexity of the smoothed variable fits. The total number of focal observations over the three years is $\mathrm{N}=151$; the subset of flowering individuals used to fit the total number of seeds of each species is given in the last row.

\begin{tabular}{|c|c|c|c|}
\hline & Dactylis & Alchemilla & Helianthemum \\
\hline $\begin{array}{l}\text { Flowering } \\
\text { probability }\end{array}$ & $\begin{array}{l}\text { 1. mean temperature } \\
\text { 2. } \text { soil moisture deficit } \\
\text { 3. minimum temperature }\end{array}$ & $\begin{array}{l}\text { 1. mean temperature } \\
\text { 2. soil moisture deficit } \\
\text { 3. GDD }\end{array}$ & $\begin{array}{l}\text { 1. mean temperature } \\
\text { 2. soil moisture deficit } \\
\text { 3. GDD } \\
\text { 4. minimum temperature }\end{array}$ \\
\hline $\begin{array}{l}\text { AIC: } \\
\text { edf } \\
\mathrm{N}\end{array}$ & $\begin{array}{l}150.36 \\
5.11 \\
151\end{array}$ & $\begin{array}{l}189.57 \\
4.02 \\
151\end{array}$ & $\begin{array}{l}154.03 \\
6.53 \\
151\end{array}$ \\
\hline $\begin{array}{c}\text { Number of } \\
\text { seeds }\end{array}$ & $\begin{array}{l}\text { 1. mean temperature } \\
\text { 2. soil moisture deficit } \\
\text { 3. GDD }\end{array}$ & $\begin{array}{l}\text { 1. mean temperature } \\
\text { 2. } \text { soil moisture deficit } \\
\text { 3. GDD }\end{array}$ & $\begin{array}{l}\text { 1. mean temperature } \\
\text { 2. } \text { soil moisture deficit } \\
\text { 3. GDD } \\
\text { 4. minimum temperature }\end{array}$ \\
\hline AIC: & 178.68 & 833.03 & 563.24 \\
\hline edf & 4.91 & 5.62 & 4.56 \\
\hline $\mathrm{N}$ & 35 & 98 & 73 \\
\hline
\end{tabular}


Figure 1. Species' low-density growth rates (LGR; in units of offspring per year per individual) given current and projected intrinsic ranges for two IPCC RCP forecasts of climate change. The two IPCC scenarios bookend change based on the most extreme (RCP 8.5, first column) and most mild (RCP 2.6, second column). Colors correspond to focal plant species (black= Dactylis glomerata $;$ red $=$ Alchemilla xanthochlora $;$ blue $=$ Helianthemum nummularium $).(\mathrm{a}-\mathrm{b})$ LGR for current year, +30 years and +60 years when only their intrinsic growth rates in the absence of competition are factored into population growth rates (with $95 \%$ prediction intervals). (c-d) Intrinsic ranges were spatially interpolated from best-fit general additive mixed models (GAMMs) of species' per-capita seed production in the absence of competitors at five sites, and then projected to future climate conditions using the same species-specific GAMMs (shaded regions are 95\% PIs). (e-f) Species' LGRs (with 95\% PIs) incorporating the full population dynamics including competition, dispersal limitation, and survival.

664

Figure 2. Climate change influences on different persistence mechanisms. Effects of climate on the benefit of competitor aggregation on the y-axis and the benefit of competitor segregation on the $\mathrm{x}$ axis for the persistence of Dactylis (black), Alchemilla (red), and Helianthemum (blue). Each arrow connects the point showing the strength of the two persistence mechanisms in the current environment to the point showing the strength of these mechanisms given the specified increase in mean temperature. The change in spatial persistence mechanisms is shown after 30 (a-b) and 60 (cd) of climate change. Vertical and horizontal gray bars are $95 \%$ prediction intervals.

Figure 3. The effect of dispersal limitation and limited population spread rates on species lowdensity growth rates (LGR; in units of offspring per year per individual) per 20-year interval with best (RCP 2.6) and worst-case (RCP 8.5) IPCC climate change scenarios. Bar groupings along the $x$-axis correspond to growth over years 2020-2040, 2040-2060, and 2060-2080. Rows and colors correspond to focal plant species: $(\mathrm{a}-\mathrm{b})$ black $=$ Dactylis glomerata $;(\mathrm{c}-\mathrm{d}) \mathrm{red}=$ Alchemilla xanthochlora; $(\mathrm{e}-\mathrm{f})$ blue $=$ Helianthemum nummularium. The darkest shading shows LGRs calculated when plants disperse globally, intermediate shading when dispersal is limited but spread rates are not, and light shading when dispersal and population spread rates are limited. 
RCP 8.5

RCP 2.6

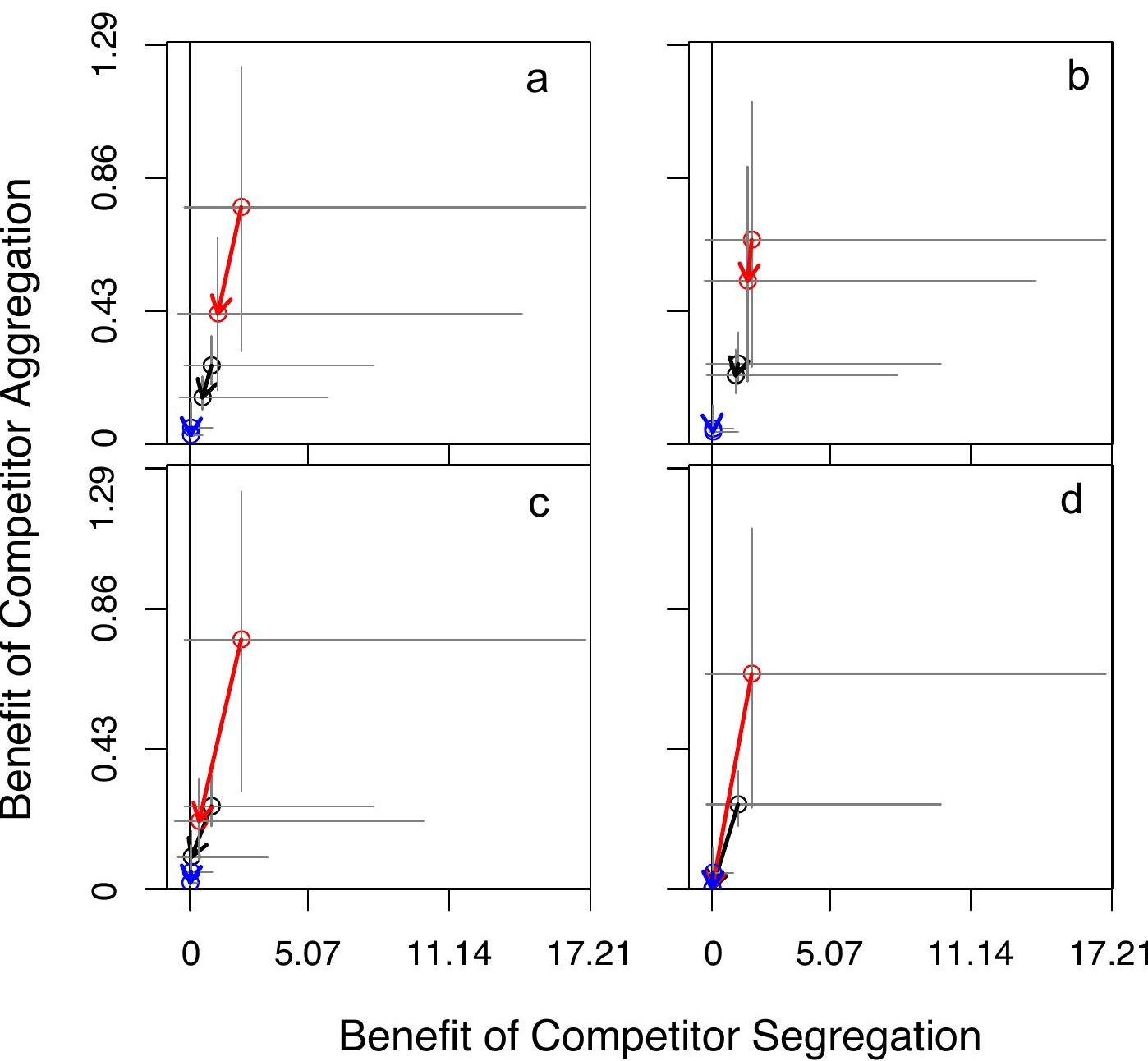


years into the future

global dispersal

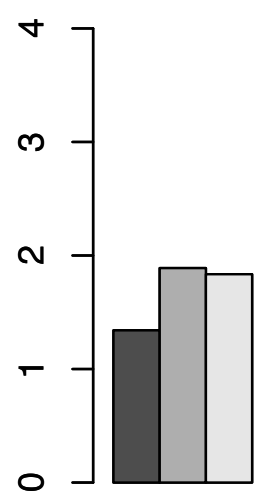

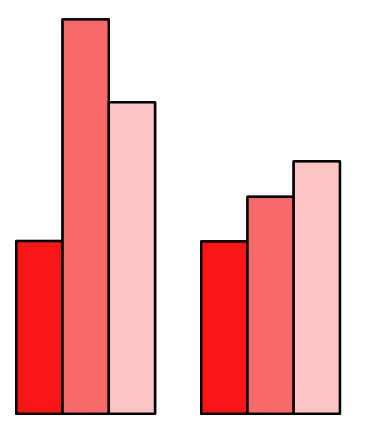

e

60
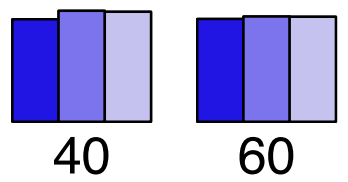

40

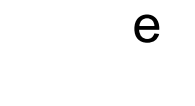

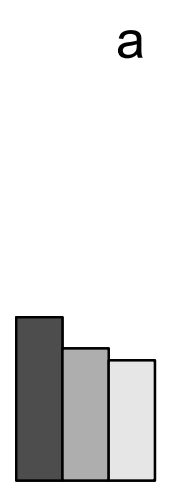

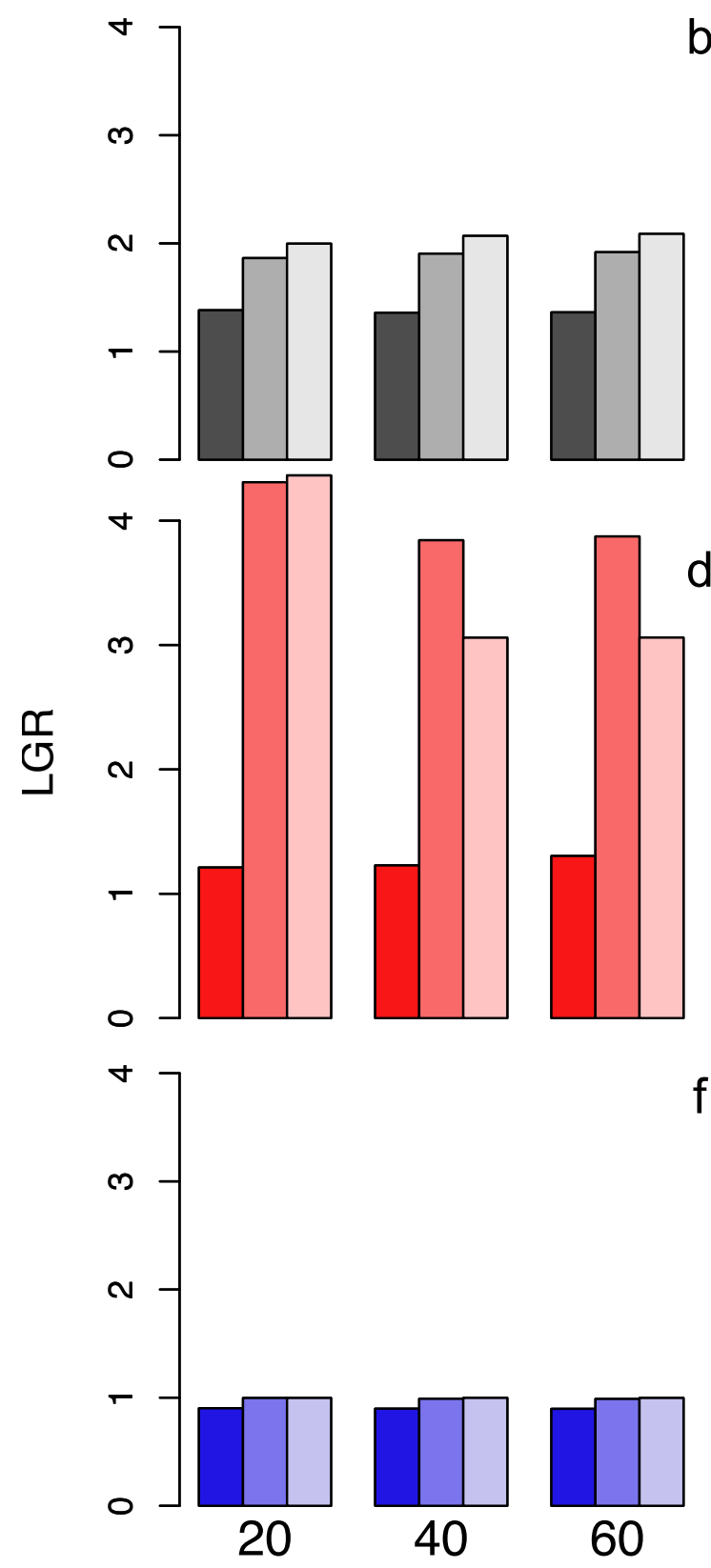

C

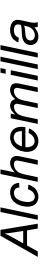

$\frac{\pi}{0}$ 
Running head: Climate-driven range shifts reduce persistence

References

Adler, P. B., S. P. Ellner, and J. M. Levine. 2010. Coexistence of perennial plants: an embarrassment of niches. Ecology letters 13:1019-1029.

Alexander, J. M., Chalmandrier, L., Lenoir, J., Burgess, T. I., Essl, F., Haider, S., ... Pellissier, L. (2018). Lags in the response of mountain plant communities to climate change. Global Change Biology, 24(2), 563-579.

Alexander, J. M., Diez, J. M., Hart, S. P., \& Levine, J. M. (2016). When Climate Reshuffles Competitors: A Call for Experimental Macroecology. Trends in Ecology \& Evolution.

Alexander, J. M., Diez, J. M., \& Levine, J. M. (2015). Novel competitors shape species/'responses to climate change. Nature. Retrieved from

Austin, M. P., \& Van Niel, K. P. (2011). Improving species distribution models for climate change studies: variable selection and scale. Journal of Biogeography, 38(1), 1-8.

Bertness, M. D. (1991). Interspecific interactions among high marsh perennials in a New England salt marsh. Ecology, 72(1), 125-137.

Bolker, B. M., Pacala, S. W., \& Neuhauser, C. (2003). Spatial dynamics in model plant communities: What do we really know? The American Naturalist, 162(2), 135-148.

Borawska-Jarmulowicz, B. (2018). Relationships between different morphological traits of panicles and seeds of Dactylis glomerata L. varieties. Agricultural and Food Science, 27(3), 179-189.

Brooker, R. W., Travis, J. M. J., Clark, E. J., \& Dytham, C. (2007). Modelling species’ range shifts in a changing climate: The impacts of biotic interactions, dispersal distance and the rate of climate change. Journal of Theoretical Biology, 245(1), 59-65.

Chen, I.-C., Hill, J. K., Ohlemüller, R., Roy, D. B., \& Thomas, C. D. (2011). Rapid Range Shifts of Species Associated with High Levels of Climate Warming. Science, 333(6045), 1024-1026.

Chesson, P. (2000). Mechanisms of maintenance of species. Annu. Rev. Ecol. Syst, 31, 343-366.

Chesson, Peter. (2000). General Theory of Competitive Coexistence in Spatially-Varying Environments. Theoretical Population Biology, 58(3), 211-237

Connell, J. H. (1961). The influence of interspecific competition and other factors on the distribution of the barnacle Chthamalus stellatus. Ecology, 42(4), 710-723. 
Davis, A. J., Jenkinson, L. S., Lawton, J. H., Shorrocks, B., Wood, S., \& others. (1998). Making mistakes when predicting shifts in species range in response to global warming. Nature, 391(6669), 783-786.

Engler, R., Randin, C. F., Thuiller, W., Dullinger, S., Zimmermann, N. E., Araújo, M. B., ... Guisan, A. (2011). 21st century climate change threatens mountain flora unequally across Europe. Global Change Biology, 17(7), 2330-2341.

Engler, R., Randin, C. F., Vittoz, P., Czáka, T., Beniston, M., Zimmermann, N. E., \& Guisan, A. (2009). Predicting future distributions of mountain plants under climate change: does dispersal capacity matter? Ecography, 32(1), 34-45.

Ettinger, A., \& HilleRisLambers, J. (2017). Competition and facilitation may lead to asymmetric range shift dynamics with climate change. Global Change Biology, 23(9), 3921-3933.

Ettinger, A. K., \& HilleRisLambers, J. (2013). Climate isn’t everything: Competitive interactions and variation by life stage will also affect range shifts in a warming world. American Journal of Botany.

Gilbert-Norton, L., Wilson, R., Stevens, J. R., \& Beard, K. H. (2010). A meta-analytic review of corridor effectiveness. Conservation Biology, 24(3), 660-668.

Godsoe, W., Jankowski, J., Holt, R. D., \& Gravel, D. (2017). Integrating Biogeography with Contemporary Niche Theory. Trends in Ecology \& Evolution.

Guisan, A., \& Thuiller, W. (2005). Predicting species distribution: offering more than simple habitat models. Ecology Letters, 8(9), 993-1009.

Haddad, N. M., Brudvig, L. A., Damschen, E. I., Evans, D. M., Johnson, B. L., Levey, D. J., ... Tewksbury, J. J. (2014). Potential negative ecological effects of corridors. Conservation Biology, 28(5), 1178-1187.

HilleRisLambers, J., Harsch, M. A., Ettinger, A. K., Ford, K. R., \& Theobald, E. J. (2013). How will biotic interactions influence climate change-induced range shifts? Annals of the New York Academy of Sciences, 1297(1), 112-125.

Hilty, J. A., Lidicker Jr, W. Z., \& Merenlender, A. (2012). Corridor ecology: the science and practice of linking landscapes for biodiversity conservation. Island Press.

Hoffmann, A. A., \& Parsons, P. A. (1997). Extreme environmental change and evolution. Cambridge University Press. 
IPCC, 2014: Climate Change 2014: Synthesis Report. Contribution of Working Groups I, II and III to the Fifth Assessment Report of the Intergovernmental Panel on Climate Change [Core Writing Team, R.K. Pachauri and L.A. Meyer (eds.)]. IPCC, Geneva, Switzerland, 151 pp.

Jankowski, J. E., Robinson, S. K., \& Levey, D. J. (2010). Squeezed at the top: interspecific aggression may constrain elevational ranges in tropical birds. Ecology, 91(7), 1877-1884.

Jump, A. S., Mátyás, C., \& Peñuelas, J. (2009). The altitude-for-latitude disparity in the range retractions of woody species. Trends in Ecology \& Evolution, 24(12), 694-701.

Katul, G. G., Porporato, A., Nathan, R., Siqueira, M., Soons, M. B., Poggi, D., ... Levin, S. A. (2005). Mechanistic Analytical Models for Long-Distance Seed Dispersal by Wind. The American Naturalist, 166(3), 368-381.

Kleyer, M., Bekker, R. M., Knevel, I. C., Bakker, J. P., Thompson, K., Sonnenschein, M., Poschlod, P., Van Groenendael, J. M., Klimeš, L., \& Klimešová, J. (2008). The LEDA Traitbase: a database of life-history traits of the Northwest European flora. Journal of Ecology, 96(6), 1266-1274.

Kot, M., Lewis, M. A., \& van den Driessche, P. (1996). Dispersal data and the spread of invading organisms. Ecology, 77(7), 2027-2042.

Kull, K., \& Zobel, M. (1991). High species richness in an Estonian wooded meadow. Journal of Vegetation Science, 2(5), 715-718.

Lenoir, J., Gégout, J.-C., Marquet, P. A., De Ruffray, P., \& Brisse, H. (2008). A significant upward shift in plant species optimum elevation during the 20th century. Science, 320(5884), 17681771.

Lenoir, J., \& Svenning, J.-C. (2014). Climate-related range shifts - a global multidimensional synthesis and new research directions. Ecography.

Massman, W. J., \& Weil, J. C. (1999). An analytical one-dimensional second-order closure model of turbulence statistics and the Lagrangian time scale within and above plant canopies of arbitrary structure. Boundary-Layer Meteorology, 91(1), 81-107.

Neubert, M. G., \& Caswell, H. (2000). Demography and Dispersal: Calculation and Sensitivity Analysis of Invasion Speed for Structured Populations. Ecology, 81(6), 1613-1628.

Ordonez, A., Williams, J. W., \& Svenning, J.-C. (2016). Mapping climatic mechanisms likely to favour the emergence of novel communities. Nature Climate Change. Retrieved from 
Running head: Climate-driven range shifts reduce persistence

Radeloff, V. C., Williams, J. W., Bateman, B. L., Burke, K. D., Carter, S. K., Childress, E. S., ... Usinowicz, J. (2015). The rise of novelty in ecosystems. Ecological Applications, 25(8), 2051-2068.

Resasco, J., Haddad, N. M., Orrock, J. L., Shoemaker, D., Brudvig, L. A., Damschen, E. I., ... Levey, D. J. (2014). Landscape corridors can increase invasion by an exotic species and reduce diversity of native species. Ecology, 95(8), 2033-2039.

Root, T. L., Price, J. T., Hall, K. R., Schneider, S. H., Rosenzweig, C., \& Pounds, J. A. (2003). Fingerprints of global warming on wild animals and plants. Nature, 421(6918), 57-60.

Schurr, F. M., Pagel, J., Cabral, J. S., Groeneveld, J., Bykova, O., O’Hara, R. B., ... Midgley, G. F. (2012). How to understand species’ niches and range dynamics: a demographic research agenda for biogeography. Journal of Biogeography, 39(12), 2146-2162.

Sexton, J. P., McIntyre, P. J., Angert, A. L., \& Rice, K. J. (2009). Evolution and Ecology of Species Range Limits. Annual Review of Ecology, Evolution, and Systematics, 40(1), 415-436.

Snyder, R. E, \& Chesson, P. (2003). Local dispersal can facilitate coexistence in the presence of permanent spatial heterogeneity. Ecology Letters, 6(4), 301-309.

Snyder, Robin E. (2006). Multiple risk reduction mechanisms: can dormancy substitute for dispersal? Ecology Letters, 9(10), 1106-1114.

Snyder, Robin E. (2008). When does environmental variation most influence species coexistence? Theoretical Ecology, 1(3), 129-139.

Snyder, Robin E., \& Chesson, P. (2004). How the spatial scales of dispersal, competition, and environmental heterogeneity interact to affect coexistence. The American Naturalist, 164(5), $633-650$.

Stanton-Geddes, J., Tiffin, P., \& Shaw, R. G. (2012). Role of climate and competitors in limiting fitness across range edges of an annual plant. Ecology, 93(7), 1604-1613.

Su, Z., Schmugge, T., Kustas, W. P., \& Massman, W. J. (2001). An Evaluation of Two Models for Estimation of the Roughness Height for Heat Transfer between the Land Surface and the Atmosphere. Journal of Applied Meteorology, 40(11), 1933-1951.

Tormo-Molina, R., Maya-Manzano, J.-M., Silva-Palacios, I., Fernández-Rodríguez, S., \& GonzaloGarijo, Á. (2015). Flower production and phenology in Dactylis glomerata. Aerobiologia, 31(4), 469-479. 
Running head: Climate-driven range shifts reduce persistence

Thompson, P. L., \& Gonzalez, A. (2017). Dispersal governs the reorganization of ecological networks under environmental change. Nature Ecology \& Evolution, 1(6), 1-8.

Urban, M. C., Tewksbury, J. J., \& Sheldon, K. S. (2012). On a collision course: competition and dispersal differences create no-analogue communities and cause extinctions during climate change. Proceedings of the Royal Society B: Biological Sciences, 279(1735), 2072-2080.

Usinowicz, J., \& Levine, J. M. (2018). Species persistence under climate change: a geographical scale coexistence problem. Ecology Letters, 21(11), 1589-1603.

Wiens, J. A., Stralberg, D., Jongsomjit, D., Howell, C. A., \& Snyder, M. A. (2009). Niches, models, and climate change: assessing the assumptions and uncertainties. Proceedings of the National Academy of Sciences, 106(Supplement 2), 19729-19736.

Williams, J. W., Jackson, S. T., \& Kutzbach, J. E. (2007). Projected distributions of novel and disappearing climates by 2100 AD. Proceedings of the National Academy of Sciences, 104(14), 5738-5742.

Wisz, M. S., Pottier, J., Kissling, W. D., Pellissier, L., Lenoir, J., Damgaard, C. F., ... others. (2013). The role of biotic interactions in shaping distributions and realised assemblages of species: implications for species distribution modelling. Biological Reviews, 88(1), 15-30.

Zarnetske, P. L., Skelly, D. K., \& Urban, M. C. (2012). Biotic multipliers of climate change. Science, 336(6088), 1516-1518. 